C-A/AP/\#191

March 2005

\title{
Electron induced molecular desorption of the RHIC beam pipes
}

\author{
U. Iriso and W. Fischer
}

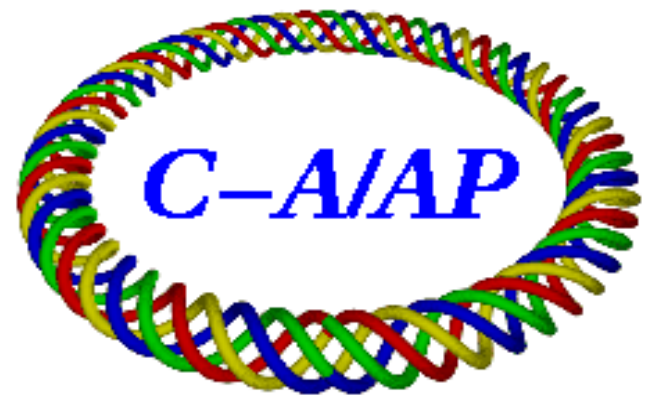

Collider-Accelerator Department Brookhaven National Laboratory Upton, NY 11973 


\title{
Electron induced molecular desorption of the RHIC beam pipes
}

\author{
U. Iriso and W. Fischer
}

Molecular desorption coefficients from electron bombardment of the warm RHIC beam pipe are derived for both baked and unbaked stainless steel. For this, we analyze electron detector and pressure gauge signals during electron clouds.

\section{Introduction}

The knowledge of molecular desorption coefficients is a key ingredient in understanding the electron cloud induced pressure rises, which are limiting machine operation in RHIC since Run-2 (2001/2002) [1-4]. RHIC was not equipped with electron detectors when the first pressure rises occurred. During the 2002 RHIC shutdown, electron detectors (ED) were installed in the RHIC ring to allow better diagnostics in the subsequent runs. In the following, we analyze the experimental data taken with these electron detectors as well as vacuum gauges during Run-3 for an unbaked surface, and Run-4 for a baked surface.

A constant electron flux into the wall $d I / d l$ is related to the pressure $P$ by

$$
P=\eta_{e} \frac{2 L k T}{e S} \frac{d I}{d l}
$$

where $(2 L)$ refers to the distance between two consecutive vacuum pumps, $S$ refers to the pumping speed for the gas specie, $k$ is Boltzman's constant and $T$ is the temperature. Finally, $\eta_{e}$ is the electron induced molecular desorption coefficient of the beam pipe wall (that is, the number of desorbed molecules per impinging electron). Since the experimental set-up does not have a Residual Gas Analyzer to investigate the pressure composition, all the calculations are done for $C O$ at room temperature [7]. The pumping speed is obtained from the manufacturers' specifications (see Table 1).

The desorption coefficient $\eta_{e}$ not only depends on the released gas species, but also on the energy of the striking electron, and the accumulated dose on the surface. For electrons below $100 \mathrm{eV}$, technical instrumentation difficulties have limited laboratory measurements, and only a small amount of data exists in the literature. This is unfortunate, since the energy of the multipacting electrons for RHIC conditions falls within this range. For stainless steel, data for energies as low as $300 \mathrm{eV}$ is found in Ref. [5], for OFHC Copper for energies as low as $20 \mathrm{eV}$ in Ref. [6].

Note that the electron flux to the wall $(d I / d l$ in Eq. 1) refers to a constant flux. However, during multipacting conditions, the electron flux is not constant, but builds up and decays within one turn. Therefore, we will be interested in the average electron flux to the wall over one turn $\left\langle\frac{d I}{d l}\right\rangle_{\tau}$, which is

$$
\left\langle\frac{d I}{d l}\right\rangle_{\tau}=\frac{1}{\tau} \int_{0}^{\tau}\left(\frac{d I}{d l}\right)_{i} d t
$$

where $\tau$ is the revolution period, and $\left(\frac{d I}{d l}\right)_{i}$ is the instantaneous flux. A flux into the wall is rewritten in terms of flux per unit area, $d I / d A$ using the beam pipe radius, $R_{p}$, as

$$
\frac{d I}{d l}=\frac{d I}{d A} 2 \pi R_{p} .
$$

This is related to the voltage in the electron detector, $V$ by

$$
\frac{d I}{d A}=\frac{V}{Z G A_{\mathrm{ED}} T_{\mathrm{eff}}}
$$

where $A_{\mathrm{ED}}$ is the area of the electron detector at the beam pipe wall, $Z$ is the line impedance, $G$ is the amplifier gain, and $T_{\text {eff }}$ is the effective transparency of the electron detector. $T_{\text {eff }}$ depends on the electron energy, and a good average value for it is $5 \%[8,9]$. In the following, the experimental electron detector signal 
is always quoted in [V]. For a given voltage and taking into account Eq. 4 and the parameters in Table 1 , the corresponding electron flux per unit area is $\frac{d I}{d A} / V \approx 3.2\left[\frac{\mu A}{\mathrm{~cm}^{2} V}\right]$.

Since we are interested in the electron flux time averaged over one turn, the ED voltage we are interested in is $\langle V\rangle_{\tau}$, which is

$$
\langle V\rangle_{\tau}=\frac{1}{\tau} \int_{0}^{\tau} V_{i} d t,
$$

where $V_{i}$ refers to the instantaneous signal in the electron detector. Typically, the ED takes a snapshot of the electron signal during one turn every 4 seconds. The signal has a time resolution of 1 ns, and it is smoothed with a $10 \mathrm{MHz}$ filter. Using Eqs. 1, 3, 4, and 5 the pressure can be expressed as a function of the electron detector voltage, $\langle V\rangle_{\tau}$, by

$$
P=\eta_{e} \frac{4 L k T \pi R_{p}}{S e G Z A_{\mathrm{ED}} T_{\mathrm{eff}}}\langle V\rangle_{\tau} .
$$

We first determine whether Eq. 6 holds for the RHIC case, and secondly we analyze the experimental data to obtain the desorption coefficient and its time evolution. The analysis focuses on two different locations, with two different surfaces, and during two different runs. The single beam vacuum chamber at "BO2" during Run-3, which was unbaked stainless steel. The common beam pipe at "IR12" during Run-4 was baked stainless steel.

\section{Electron induced desorption of unbaked stainless steel}

\subsection{Experimental set up}

During Run-3, approximately $60 \%$ of the warm RHIC beam pipes were baked. Beam injection is stopped if the pressure at any location in the ring approaches hazardous limits. These limits were approached within the $40 \%$ of the unbaked regions, where the electron multipacting thresholds are lower than in the baked regions. The pressure rises in unbaked regions prevented that multipacting conditions in baked regions were reached at the same time. In this situation, the only electron detector in an unbaked region (BO2) is easily exposed to electron clouds. Only a few electron clouds were detected in the baked regions with electron detectors because the multipacting conditions were rarely reached. Therefore, the analysis during Run-3 is focused on the detector at $\mathrm{BO} 2$.

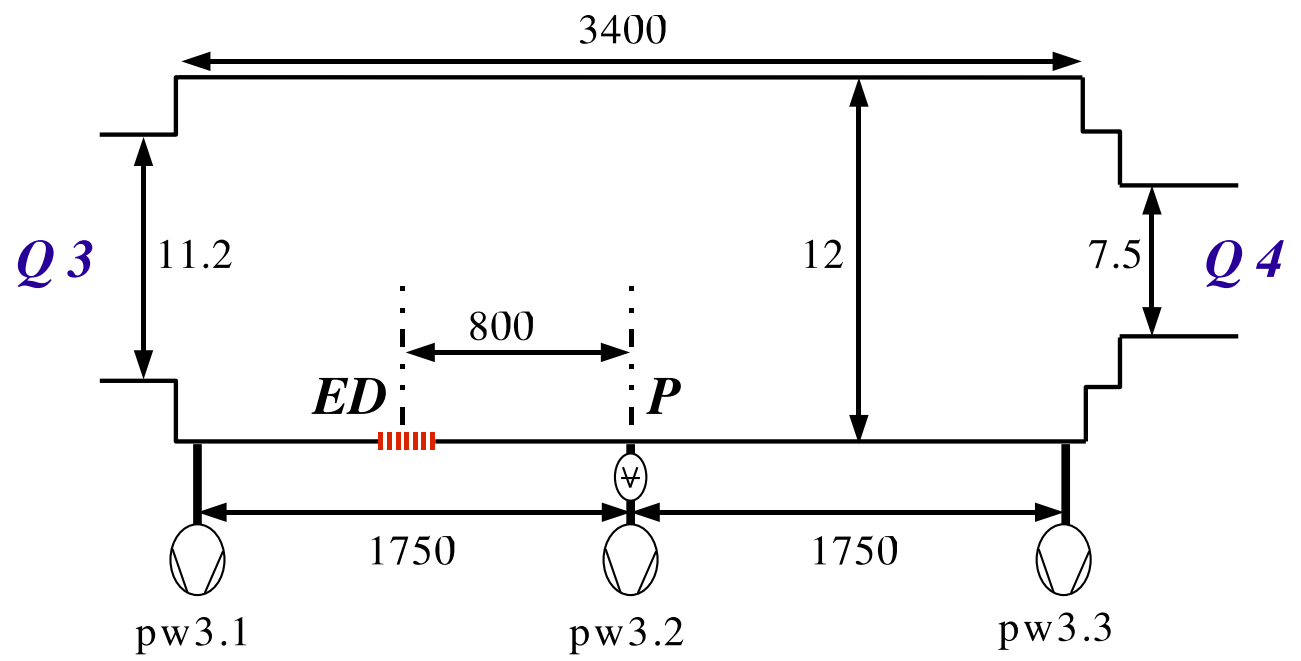

Figure 1: Geometry of the single beam pipe BO2, made of stainless steel and unbaked during Run-3. The electron detector location (marked with $E D$ ) is about $8 \mathrm{~m}$ away from the pressure gauge and vacuum ion pump, whose location is marked with $P$. Distances are given in $\mathrm{cm}$ and are not to scale.

The single beam pipe in $\mathrm{BO} 2$ is shown in Fig. 1. The vacuum pump and pressure gauge are at the same location, the electron detector is $8 \mathrm{~m}$ away. Since the beam pipe between the ED and the vacuum 
pump/gauge is made of the same material, we assume that the electron flux is the same throughout that region. Therefore, we apply Eq. 6 as if the ED were in the same location as the vacuum pump and gauge.

Table 1: Parameters used to estimate the electron desorption coefficient at BO2 and IR12.

\begin{tabular}{lcccc}
\hline \hline & & & BO2 & IR12 \\
parameter & symbol & unit & value & value \\
\hline distance between pumps & $2 L$ & $\mathrm{~m}$ & 16 & 3.25 \\
beam pipe radius & $R_{p}$ & $\mathrm{~cm}$ & 6 \\
pumping speed for $C O$ & $S$ & $1 / \mathrm{s}$ & 600 \\
impedance line & $Z$ & $\Omega$ & 50 \\
amplifier gain & $G$ & - & 1600 \\
electron detector area & $A_{\mathrm{ED}}$ & $\mathrm{cm}^{2}$ & 78 \\
effective transparency & $T_{\mathrm{eff}}$ & $\%$ & 5 \\
\hline \hline
\end{tabular}

\subsection{Data analysis in $\mathrm{BO} 2$}

Figure 2 shows an example of experimental data. The top plot shows the time evolution of the pressure at the gauge pw3.2 (red line, right vertical axis), and the electron signal averaged over one turn (black dots, left vertical axis, calculated using Eq. 5) as beam injection takes place (bottom plot). Note that injection is temporarily halted for about two minutes after 45 bunches $(12 \mathrm{~h} 12 \mathrm{~m})$. Injection resumed $(12 \mathrm{~h} 14 \mathrm{~m})$ and finally finished at $12 \mathrm{~h} 20 \mathrm{~m}$. As the beam decays, both the electron signal and the pressure signal decay at the same rate.
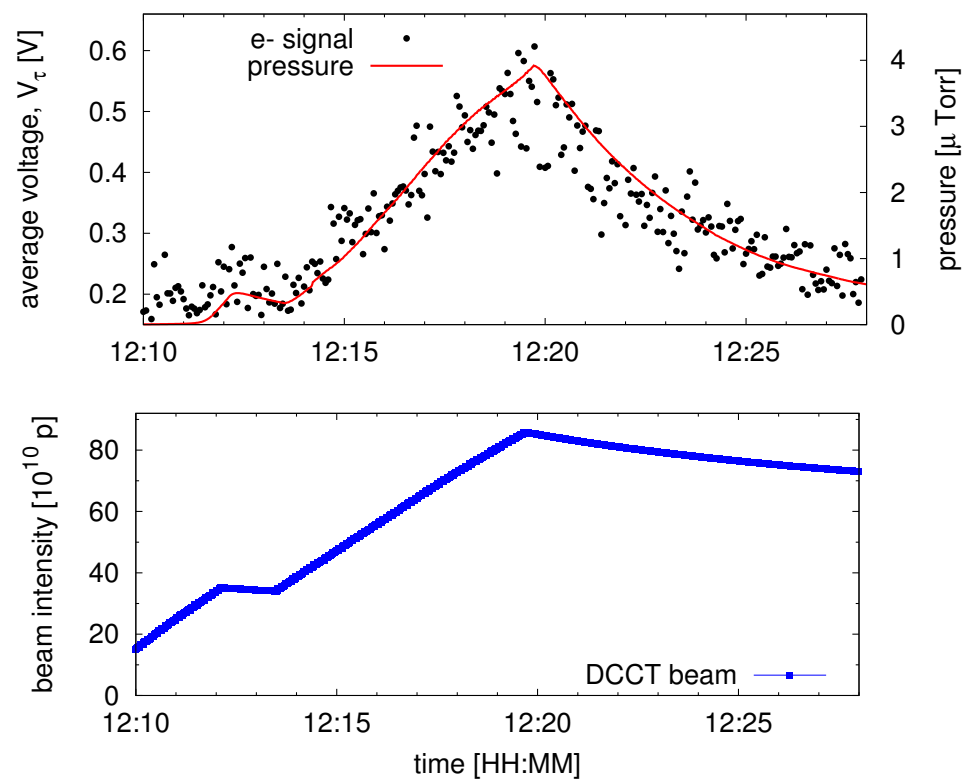

Figure 2: Dynamical pressure evolution and eletron signal (top plot), as the blue beam is being injected (bottom plot) for fill 3460. Pressure and electron signal follow a similar evolution.

A linear relation between the pressure readings with the electron signal averaged over one turn is confirmed in Fig. 3, and by the corresponding regression coefficient (0.947), which validates the initial assumption in Eq. 6. This linear relation has been found also in other accelerators $[11,12]$. The black line shows the result of a linear regression applied to the red points,

$$
P=B\langle V\rangle_{\tau}+A,
$$

where $B$ and $A$ are the fitting coefficients. The independent term is added to account for the static pressure produced by thermal outgassing, and for the electron detector noise. It then follows that the desorption 
coefficient, $\eta_{e}$ is

$$
\eta_{e}=B \frac{e S_{z} Z G A_{\mathrm{ED}} T_{\mathrm{eff}}}{4 L k T \pi R_{p}} .
$$

For the case in Fig. 2, the correlation coefficient is $R=0.947$, the error in $B$ is $2 \%$, and the desorption coefficient ( $C O$ equivalent) is $0.18 \pm 50 \%$. The error stems from the uncertainty in the pressure reading and pumping speed values [7].

We perform this analysis for all the fills during Run-3 that produced electron detector signals above the noise level. Figure 4 shows the evolution of the calculated desorption coefficient until the end of the run (fill 3812). As expected, this coefficient decreases with time due to the bombardment dose. An estimate of the total bombardment dose is difficult. The signal/noise ratio of the electron detector does not allow electron signals to be obtained under about $0.15 \mathrm{~V}$, even though pressure rises are observed.

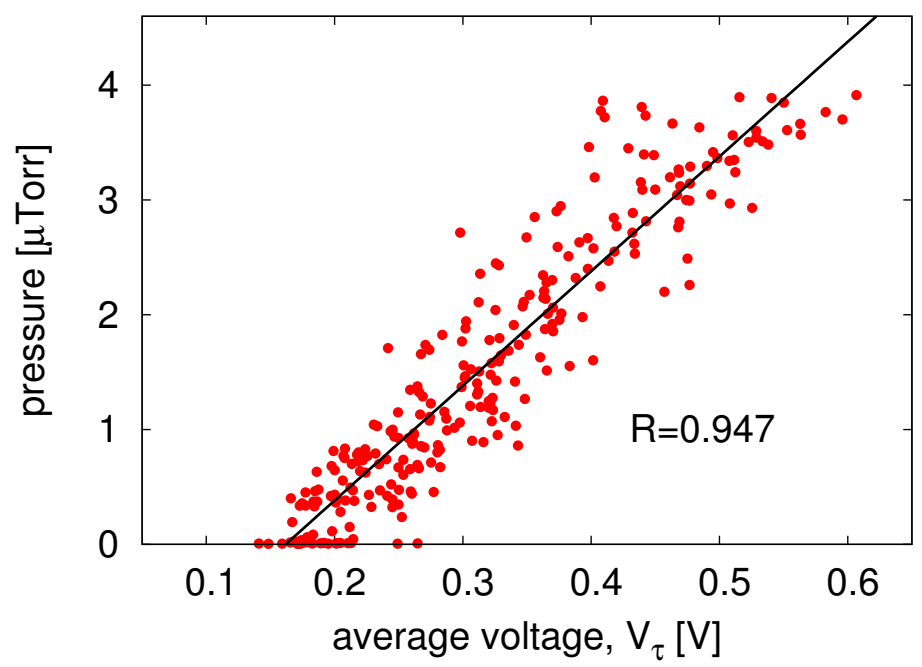

Figure 3: Linear fit (black line) to the experimental data (red points) from Fig. 2. Linear relation between the pressure and the ED voltage, $\langle V\rangle_{\tau}$ is confirmed.

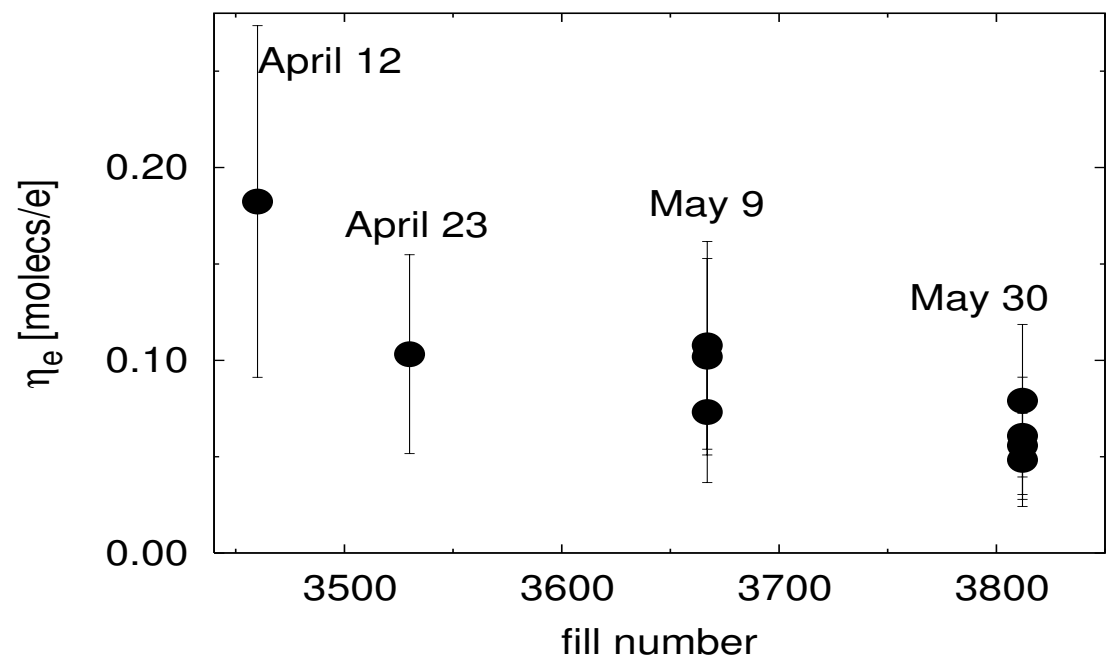

Figure 4: Summary of all calculated desorption coefficients for the unbaked surface BO2. The error bar $(50 \%)$ stems from the uncertainty in the pumping speed and vacuum pressure readings. 

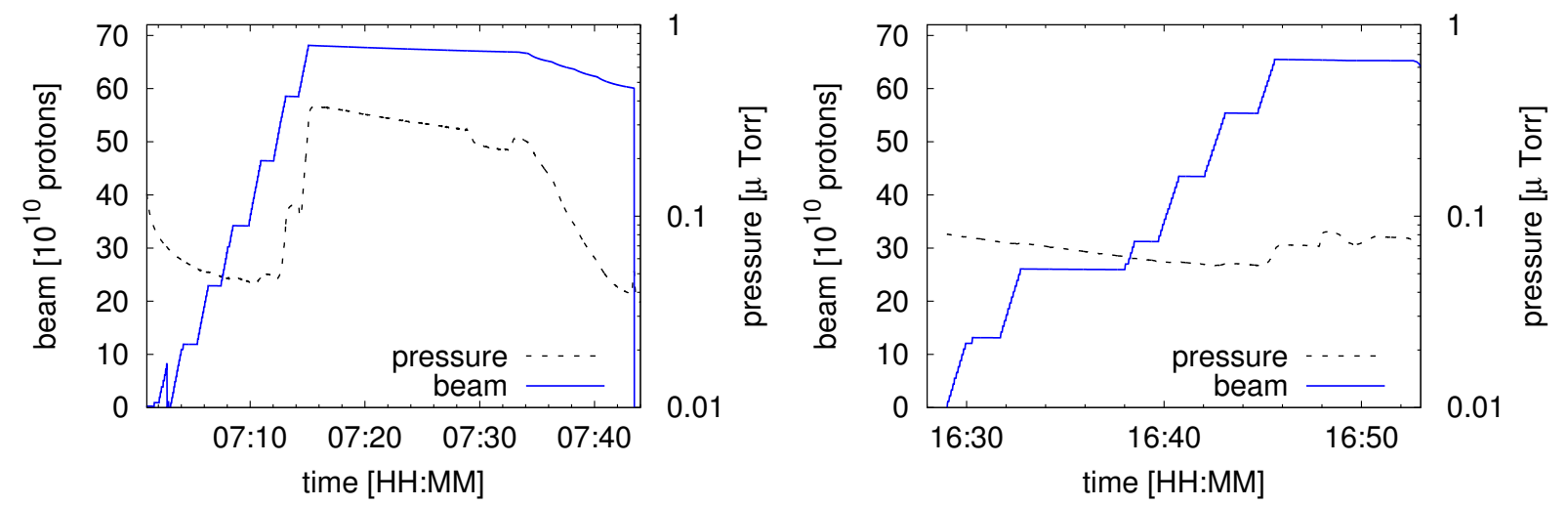

Figure 5: Two fills, five weeks apart, with the same bunch pattern, producing different pressure rises. Fill 3530 (left plot) shows how the pressure rises by about one order of magnitude. In fill 3812, with similar beam conditions, the pressure rise is not noticeable.

07:16:04

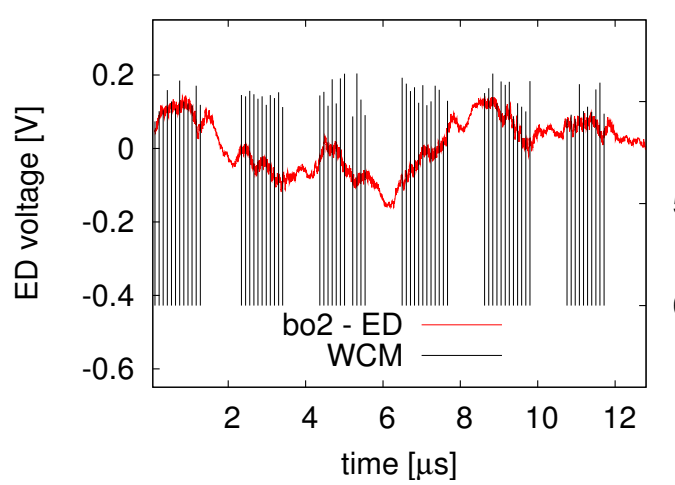

16:48:20

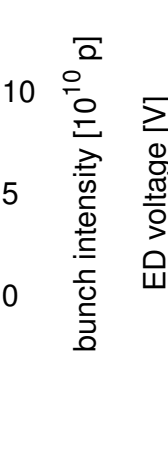

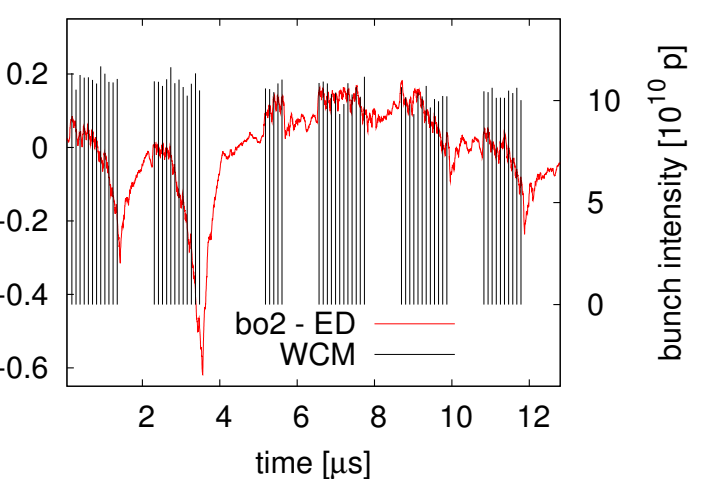

Figure 6: Electron detector snapshots for fill 3530 (left) and 3812 (right). The electron signal for fill 3530 is within the noise signal. For fill 3812, the signal is clear although fill 3530 has a pressure rise one order of magnitude larger than fill 3812 (see Fig. 5)

\subsubsection{Scrubbing and electron detector noise}

Unscrubbed unbaked surfaces easily produce large pressure rises under small electron fluxes. N. Hilleret shows in Ref. [14] two important facts needed to understand the problems with RHIC's electron detection signals. First, the decrease in electron desorption coefficient $\eta_{e}$ starts with a smaller dose than the decrease in the Secondary Emission Yield, $\delta$. Secondly, the scrubbing efficiency is better for $\eta_{e}$ than for $\delta$, or, in other words Ref. [14] shows that

$$
\left|\frac{\partial \eta_{e}}{\partial D}\right|>\left|\frac{\partial \delta}{\partial D}\right|
$$

where $D$ is the bombardment electron dose. Therefore, small signals within the ED noise can produce large pressure rises for "as received" surfaces, preventing injection of high intensity beams. As the dose increases, larger beam intensities can be injected and hence the likelihood of producing electron fluxes above the ED noise, increases.

This is illustrated in Figs. 5 and 6, which show injection for fills 3530 and 3812, using the same bunch pattern. The two injections were 5 weeks apart. The bunch train in both cases is composed of 6 batches of 12 consecutive bunches spaced by $108 \mathrm{~ns}$, followed by a gap of 864 ns. The position of the bunches is represented with vertical black bars in Fig. 6. Left plot in Fig. 5 shows the pressure as a function of the total 
beam injected in the machine for both fills (bold line for fill 3530, dotted line for fill 3812). The pressure for fill 3530 rises to a larger value than for fill 3812. However, the right plot in Fig. 6 shows an ED snapshot for each fill. Whereas no clear electron signal is observed for fill 3530 (red trace on the left hand side plot), the signal for fill 3812 clearly builds up in correlation with the bunch passages (red trace in the right hand side plot). The reason why there can be both an electron signal with no associated pressure rise, and conversely a pressure rise without a signal, can be understood by considering the different influence the scrubbing effect has on $\eta_{e}$ and $\delta$ (see above). However, we note there can be other causes for this effect. For example, an irregular longitudinal distribution of the multipacting along the vacuum chamber, or the slightly modified bunch pattern in fill 3812 .

\section{Electron induced desorption of baked stainless steel}

\subsection{Experimental setup}

Approximately $80 \%$ of the warm beam pipes in RHIC were baked by Run-4 (2003/2004), including the aforementioned section BO2. During the polarized proton run, electron clouds were often detected in the common beam pipe, "IR12". Two beams in opposite directions traversing a common beam pipe produce either larger bunch intensities (where both beams coincide) than in the single beam pipes, or shorter bunch spacings than the ones in the single beam pipes. Therefore, electron cloud thresholds in the common beam pipe regions are easier to reach than in single beam pipes.

Figure 7 shows the experimental set up in IR12. Note that the ED, vacuum pump, and pressure gauge are only $0.3 \mathrm{~m}$ apart. About $3 \mathrm{~m}$ away from the ED there are $5 \mathrm{~m}$ of NEG coated beam pipe. The same method as in Section 2.2 is applied to analyze the data: we assume that ED, vacuum pump and gauge are at the same location.

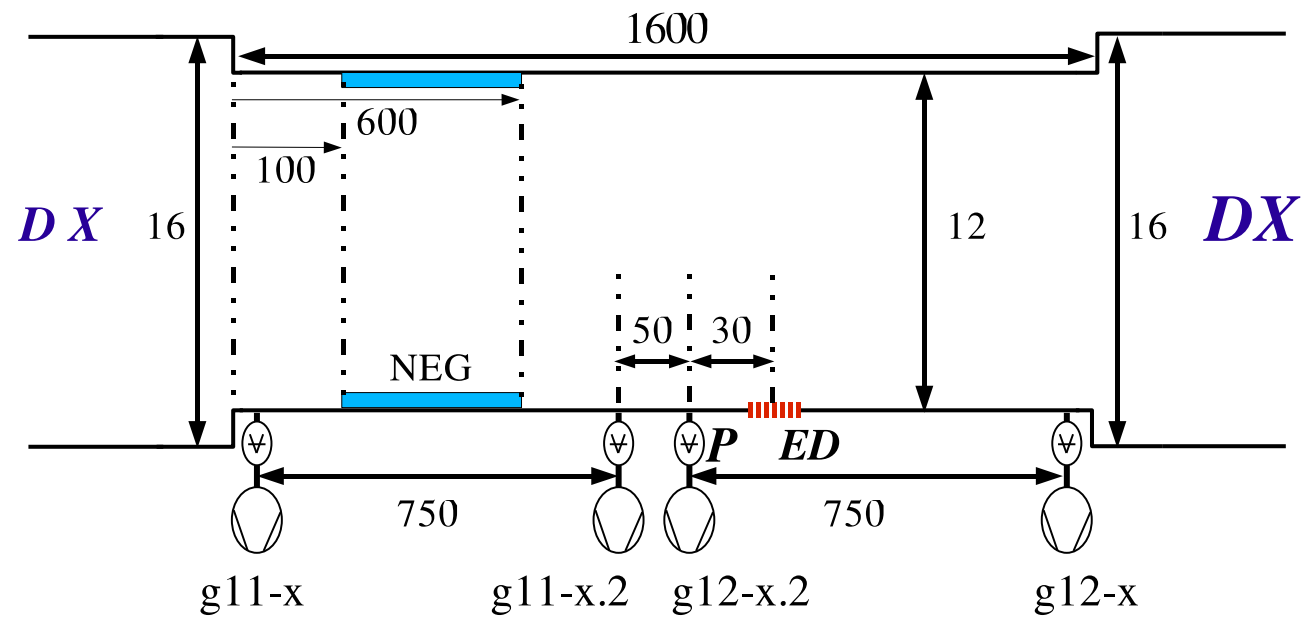

Figure 7: Geometry of the common beam pipe IR12, made of baked stainless steel. The electron detector location (marked with $E D$ ) is only $0.3 \mathrm{~m}$ away from the pressure gauge and vacuum ion pump, whose location is marked withe $P$. Distances are given in $\mathrm{cm}$ and are not to scale.

\subsection{Data analysis in IR12}

An example of an electron cloud, and its correlation with the pressure in IR12 is shown in Fig. 8. Once injection into the blue ring has finished (blue line, bottom plot), the electron cloud is triggered after approximately 36 bunches are injected in the yellow ring (red line, bottom plot), and both the pressure (red trace, top plot) and the electron signal (black points, top plot) start increasing at the same rate. Note the "swing" in both the electron signal and the pressure evolution (top plot), as the bunch length (denoted with blue and red squares for the blue and yellow beam, respectively) swings during the ramp (bottom plot). Shorter bunches produce electrons with larger striking energies at the wall, which translates into a larger secondary emission yield and a larger flux into the wall. 

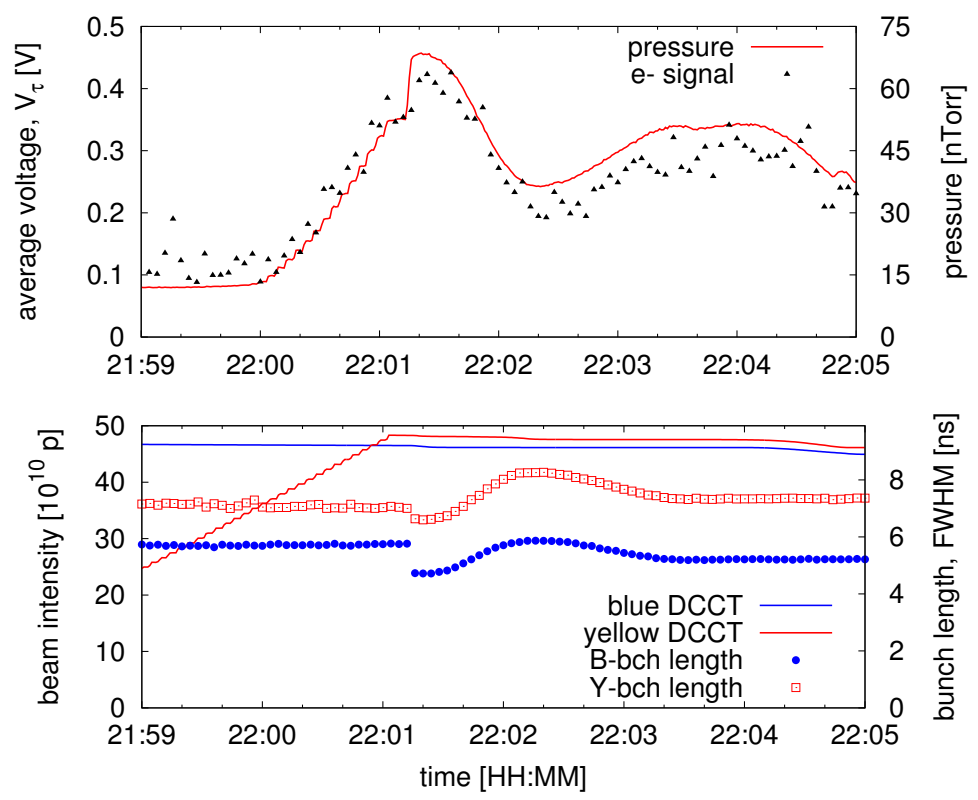

Figure 8: Dynamical evolution of the pressure rise, and eletron signal (top plot), as the blue beam is being injected (bottom plot) for fill 5201. At the beginning of the energy ramp (about 22:01:20) the rf voltage is raised leading to shorter bunches.

Again, the correlation between pressure and electron detection is confirmed when plotting the electron detector voltage and the pressure (see Fig. 9). For the particular case in Fig. 9, the calculated desorption coefficient is $\eta_{e}=0.007 \pm 50 \%, \mathrm{R}=0.942$, and the error in $\mathrm{B}$ is $3.7 \%$.

Figure 10 shows the analysis for all the fills producing electron signals in IR12 during Run- 4 . Note that no scrubbing effect is seen. It is possible that the chamber is sufficiently conditioned by baking, and/or the low energy of the multipacting electrons do not affect the surface status. This could also be due to the presence of the hydrogen jet installed to measure beam polarization. The jet injects an extra gas load for several minutes to measure the beam polarization [15]. If one understands the scrubbing as a "cleaning" effect, the injection of gas can cancel this effect. For these reasons, we think the numbers that are useful for describing the desorption coefficient for a baked surface are the average value, and the standard deviation of the measurements in Fig. 10: $0.008 \pm 0.003$.

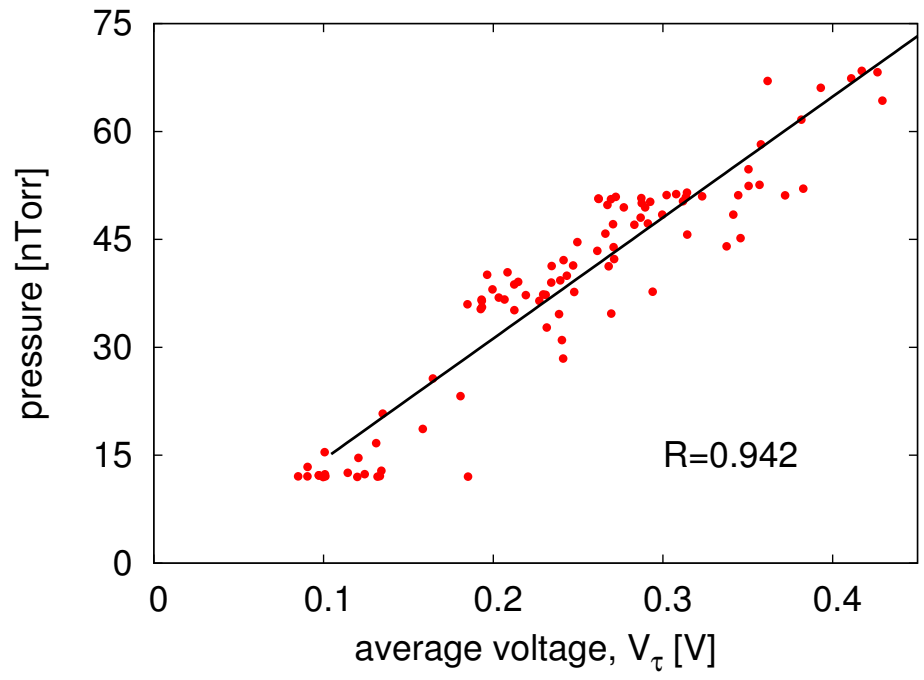

Figure 9: Linear relation between the electron signal and the pressure for fill \#5201 at IR12. 


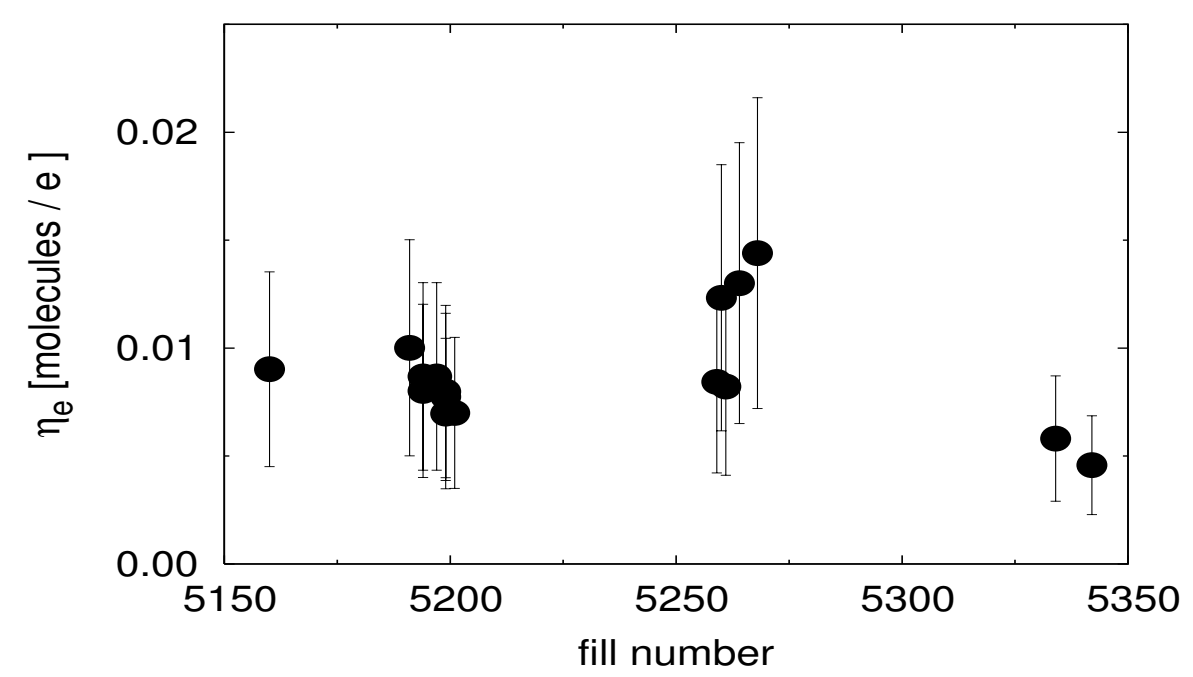

Figure 10: Summary of all calculated desorption coefficients for the baked staineless steel furface at IR12 during Run- 4 . The error bar is about a $50 \%$ of the calculated value due to uncertainty in the pumping speed and vacuum pressure readings. The average and rms value are $0.008 \pm 0.003$.

\section{Electron energy during multipacting conditions}

The desorption coefficient strongly depends on the striking electron energy at the beam-pipe wall. As mentioned earlier, instrumental limitations arise when trying to obtain laboratory measurements for the desorption coefficient at low electron energies.

The electron energy spectrum was measured during fill \#3812 at BO2 with the equipment shown in Ref. [9]. Using the Retarding-Field Analyzer electron detector, several energy sweeps were carried out during fill 3812. Figure 11 shows the absolute value of the ED voltage for two different energy sweeps (red and blue curves), and the electron energy distribution (black line), given by the derivative of both curves. Note that the oscillations in the sweeps produce as well oscillation in the spectrum. The two main features are the large peak of low energy electrons $(15 \mathrm{eV})$, and a spectrum extending to about $350 \mathrm{eV}$. Since the noise in the ED is around $0.15 \mathrm{~V}$, we cannot definitely conclude the upper energy limit for the energy spectrum.

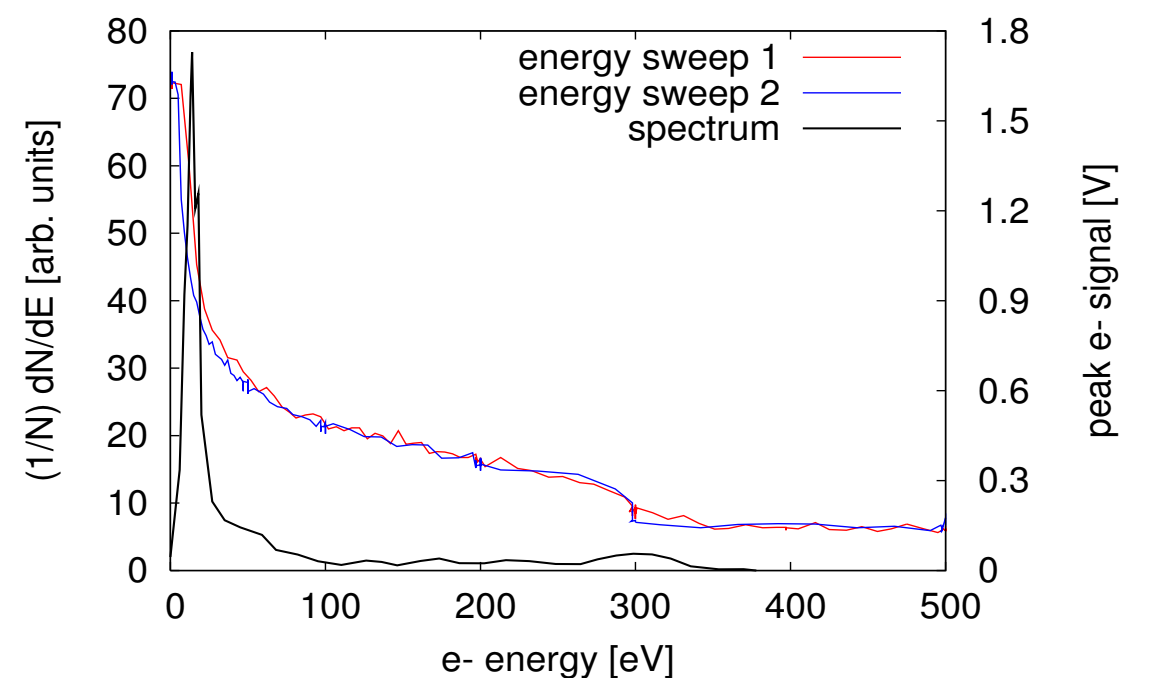

Figure 11: Two measured integrated energy spectrum (red and blue traces), and the derived electron distribution for energies between 0 and $500 \mathrm{eV}$. 


\section{Summary}

A linear relation between the pressure evolution and the electron flux into the wall due to an electron cloud is confirmed. The electron desorption coefficient $\eta_{e}$ is inferred from the analysis of the experimental data. For unbaked stainless steel and assuming $C O$ as the only desorbed gas, this value is 0.18 at the beginning of the run, and decreases to 0.05 after 5 weeks of machine operation due to scrubbing. For baked stainless steel, this value is around 0.008 , and no scrubbing effect is noticeable. For unbaked stainless steel, a measure of the energy spectrum shows a large peak around $15 \mathrm{eV}$. The spectrum extends to at least $350 \mathrm{eV}$.

\section{Acknowledgements}

We are indebted to M. Blaskiewicz for pointing subtile features of the electron detector signals, and to H.C. Hseuh, who patiently provided all vacuum related parameters. We are also thankful to H. Huang, R.C. Lee, L. Smart, V. Ptitsyn, and S.Y. Zhang for discussions and help.

\section{References}

[1] W. Fischer et al. Vacuum pressure rise with intense ion beams in RHIC. Proceedings of EPAC'02, Paris, 2002.

[2] S.Y. Zhang. RHIC Vacuum Pressure Bump. BNL C-A/AP/67, 2002.

[3] H. Hseuh, L. Smart, S.Y. Zhang. Analysis of beam induced pressure increases in RHIC warm vacuum sections. Proceedings of EPAC'02, Paris, 2002.

[4] W. Fischer, M. Blaskiewicz, P. He, H. Huang, H.C. Hseuh, U. Iriso, G. Rumolo, L. Smart, D. Trbojevic, and S.Y. Zhang. Electron clouds and vacuum pressure rises at RHIC. Proceedings of ECLOUD'04, Napa Valley (USA), April 2004.

[5] J. Gomez-Goni and A.G. Mathewson. "Temperature dependence of the electron induced gas desorption yields on stainless steel, copper and aluminum". J. Vac. Sci. Technol. A 15 (6), Nov/Dec 1997.

[6] F. Billard, N. Hilleret, G. Vorlaufer, "Some results on the electron induced desorption yield of OFHC copper", CERN $\mathrm{LHC} / \mathrm{VAC} / \mathrm{FB} / \mathrm{NH} / \mathrm{GV} 00-32(2000)$.

[7] H.C. Hseuh, private communications.

[8] P. He et al, Calibration of the RHIC electron detector. Proceedings of PAC'03, Portland, 2003.

[9] U. Iriso, A.K. Drees, W. Fischer, D. Gassner, O. Gould, J. Gullotta, P. He, H.C. Hseuh, R. Lee, V. Ponnaiyan, L. Smart, D. Trbojevic, and S.Y. Zhang. Electron cloud observations at RHIC in Run-3 (2002/2003). CAD/AP/129, Dec. 2003.

[10] W. Fischer, M. Blaskiewicz, M. Brennan, T. Satogata, Electron cloud measurements and simulations for the Brookhaven Relativistic Heavy Ion Collider, PRST-AB 5, 124401 (2002).

[11] K. Kanazawa and H. Hisamatsu, Electron Cloud measurement at KEKB. Presentation at BNL, October 2004.

[12] Y. Suetsugu. Observation and simulation of the nonlinear dependence of vacuum pressures on the positron beam current at the KEKb. Proceedings of PAC'01, June 2001.

[13] Proceedings of CERN Accelerator School on Vacuum Technology, held at Snekersten, Denmark. Ed. by S. Turner in Geneva, 1999.

[14] N. Hilleret, B. Henrist, C. Scheuerlein, M. Taborelli, G. Vorlaufer. The Variation of the Secondary Electron Yield and of the Desorption Yield of Copper under Electron Bombardment: Origin and Impact on the Conditioning of LHC, Proceedings of EPAC'02, 2553, June 2002.

[15] A. Zelenski, A. Bravar, D. Graham, W. Haeberli, S. Kokhanovski, Y. Makdisi, G. Mahler, A. Nass, J. Ritter, T. Wise, V. Zubets. Absolute Polarized H-Jet polarimeter development for RHIC, PST 2003 Workshop on polarized sources and targets, Novosibirsk, (Ru). PBD: 22 Sep 2003 